\title{
Multimodal face recognition method with two-dimensional hidden Markov model
}

\author{
J. BOBULSKI \\ Institute of Computer and Information Science, Czestochowa University of Technology, 73 Dabrowskiego St., 42-201 Czestochowa, Poland
}

\begin{abstract}
The paper presents a new solution for the face recognition based on two-dimensional hidden Markov models. The traditional HMM uses one-dimensional data vectors, which is a drawback in the case of $2 \mathrm{D}$ and $3 \mathrm{D}$ image processing, because part of the information is lost during the conversion to one-dimensional features vector. The paper presents a concept of the full ergodic 2DHMM, which can be used in 2D and $3 \mathrm{D}$ face recognition. The experimental results demonstrate that the system based on two dimensional hidden Markov models is able to achieve a good recognition rate for $2 \mathrm{D}, 3 \mathrm{D}$ and multimodal $(2 \mathrm{D}+3 \mathrm{D})$ face images recognition, and is faster than ICP method.
\end{abstract}

Key words: pattern recognition, biometrics, 3D face recognition, hidden Markov model.

\section{Introduction}

Automatic face recognition techniques are a challenging problem which has received attention due to its potential applications in different fields such as security applications, access control or video indexing. There is no robust technique that provides a solution to all situations and different applications that face recognition may encounter. Face recognition has its own peculiar group of problems related to creating automatic systems, like lighting differences, pose and expression variations, and resolution of the image acquisitions [1]. Illumination or pose can change the appearance of a face significantly, and in most cases the differences induced by lighting or pose variations are larger than differences between individuals, which makes the recognition task very difficult. Hence, problems caused by lighting and pose variations are the main factors contributing to the degradation of 2D face recognition algorithms [2]. In last years, some of the new face recognition strategies have tended to overcome face recognition problem from a 3D perspective [3]. The 3D data points proper to the surface of the face could give us more useful information for recognition, theoretically, and solve the problem of pose and lighting variations. However, 3D face recognition is linked to other difficulties, e.g. normalization, devices for acquiring faces, time and cost [4].

In the literature, many useful reviews of 3D face recognition problem may be found, e.g. made by Abate et al. [5] or Bowyer et al. [6]. The 3D face recognition approaches using range images can be categorized into three classes: (1) PCAbased approaches, (2) feature-based approaches, and (3) surface matching approaches $[4,6]$. In the first class, principal component analysis (PCA) is applied to range data to reduce the dimension and then the recognition is performed by matching a probe image with gallery images in a lower dimension space. These approaches are simple and fast, but they have low efficiency rate compared to the other approaches [12-15]. In the

*e-mail: januszb@icis.pcz.pl second class, imaging performance at specific points to a collection wavelet filter is calculated and treated as a set of features. Next, based on the result of the comparison of these collections a decision is made about the recognition [10, 16-18]. These methods have high performance rate compared to approaches in the other two class, but they use the 2D images for landmark localization, as it is critical for these methods. In the last class, researchers use the iterative closest point (ICP) or Hausdorff distance (HD) to adjust the 3D surface points of a face and then realize the recognition based on the minimum distance between the two faces $[8,9]$. These methods have high recognition rate, but their main problem is speed and computational complexity. For 3D face recognition from range data, Gordon used Gaussian curvature [16] to extract facial features used in recognition process. Acherman et al. [13] applied the Eigenface and hidden Markov model (HMM) for recognition. Pan et al. [12] used both the partial directed HD and a PCA-based method for 3D face recognition. Lu et al. [11] reported a system that combines surface matching using ICP - and appearance-based matching. Tanaka et al. [17] presented a curvature-based approach for range image segmentation and represented the face by extended Gaussian image. Another method was proposed by Jin et al. [19]. They use ICP, Gabor wavelet transform and local binary pattern (LBP), which offers very good results, but this method is computationally expensive. Bicego et al. [27]propose 1D-HMM and wavelets for face recognition with very good recognition rate, but only for $2 \mathrm{D}$ images and the experiment was made with a small database (40 subjects).

Many methods arose for 3D face recognition, but most of them, except for [13, 26, 28, 20], did not attempt to use HMM. It may result from the fact that traditionally, HMM was used for one-dimensional features vectors, which is a crucial problem in the $3 \mathrm{D}$ data processing. The classical one-dimensional HMM processes one-dimensional data, which is impractical in image processing, because the images have two dimensions. We lose some information about the contents when we convert an image from 2D to 1D. So we should apply two-dimensional HMM if we process two-dimensional data, and 2DHMM should work 
with 2D data. One possibility is a pseudo-2D HMM [21, 22] or embedded HMM [28], which is an extension of classic 1DHMM. They have super-states covering one-dimensional hidden Markov models. So, we have 1D model with 1D data in practice. Paper [23] presents analytic solution and proof of correctness for two-dimensional HMM, which is similar to MRF [24-26], but works with one-dimensional data. Additionally, it can be applied only for left-right type of HMM.

That is the main reason why we decided to develop a 3D facial recognition method using two-dimensional HMM, which processes two-dimensional data. As with 1D HMM, the most important thing for 2DHMM is to solve two basic problems, namely the probability evolution and parameter estimation. Moreover, the presented algorithms are implemented for ergodic models, instead of left-right type [23]. In this paper we focus on the face recognition method in 2D and 3D images with two-dimensional hidden Markov models (2DHMM), using wavelets for feature extraction. The paper is organized as follows: first the pre-processing procedure needed to obtain the pure face image is described; next we describe feature extraction; then we present the algorithms for two-dimensional data in HMM; afterwards, experiments are performed on the facial database with different experimental conditions. Conclusions are given in the last section.

\section{Proposed method}

2.1. Scheme of the proposed method. The structure of proposed method is shown in Fig. 1.

2.2. Pre-processing procedure. Pre-processing is a very important part of a persons' identification process and the ideal output of this procedure is to obtain a face image showing the entire face in vertical pose, with normalized intensity and equal size. Furthermore, at this stage illumination and lighting effects should be removed. The majority of these conditions is fulfilled by the UMB-DB database [35] used in the experiments. Our system includes a pre-processing procedure which is composed of three steps: 1) locating and cropping the face region using a rectangle according to face shape; 2) selecting face area; 3 ) scaling the image so that the distance between the inner corners of the eyes is equal 120 pixels. The detailed description of this procedure is provided in [29]. The database delivers coordinates of the eyes inner corners. Based on these points, the face image is scaled so that the distance between them is equal to 120 pixels. Then the main area of the face is selected and the areas containing little useful information, namely the forehead, ears and chin are removed (Fig. 2).

2.3. Feature extraction with wavelet transform. The discrete wavelet transform (DWT) is a method widely used for imaging systems, because it can be customized to a signal by choosing
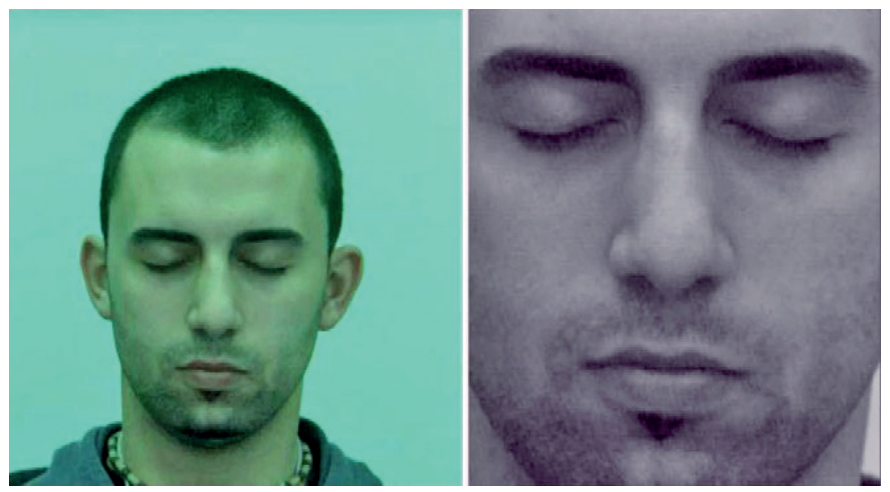

Fig. 2. The effect of pre-processing procedure
Learnig

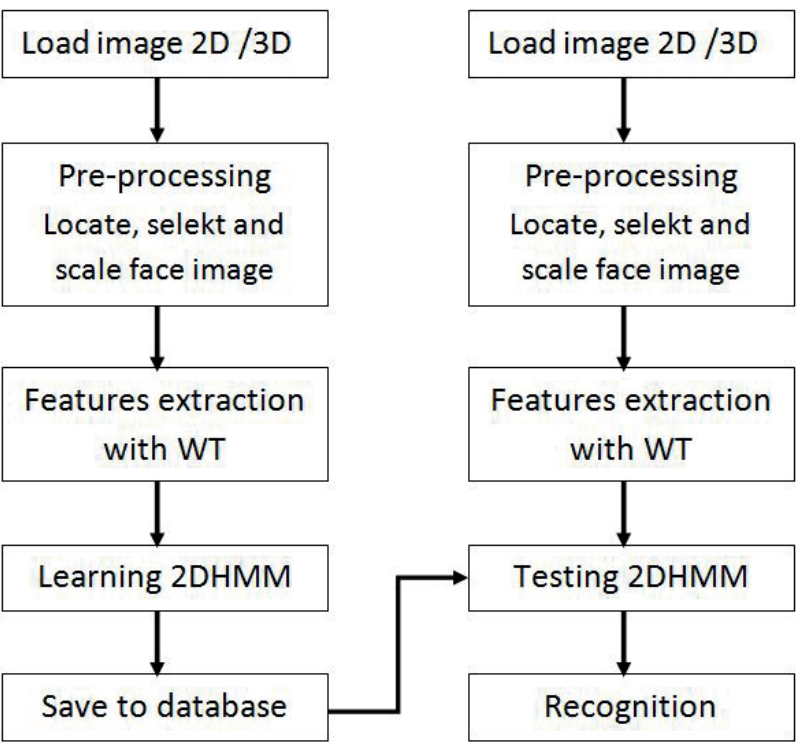

Testing
Fig. 1. Scheme of proposed method proper wavelet function. DWT decomposes the signals into subbands with both time and frequency information and provides signal features. Additionally, a major advantage of DWT is the ability to perform local analysis, that is, to analyse a localized area of a larger signal. A detailed description of the well-known one-dimensional DWT can be found in [30].

One-dimensional wavelet transform is defined as the convolution of signal and wavelet function, shifted in time and with scaling wavelet function. As a result we obtain approximations and signal details, with the approximations being highscale low-frequency components and the details - the low-scale high-frequency components of the signal. Congruous decomposition process may be used for two- or three-dimensional signals. The three-dimensional wavelets may be considered as submission of 1D wavelets by applying a 1-D wavelet decomposition in three directions $x, y, z$. Fig. 3 presents a one-level 3D discrete wavelet decomposition of a $3 \mathrm{D}$ image. $\mathrm{L}$ and $\mathrm{H}$ denote the low and high frequency components under low and high frequency filters, respectively. The image is first filtered along the columns, resulting in a low-pass image and a high-pass image. Both $\mathrm{L}$ and $\mathrm{H}$ are then filtered along the rows, resulting in four 


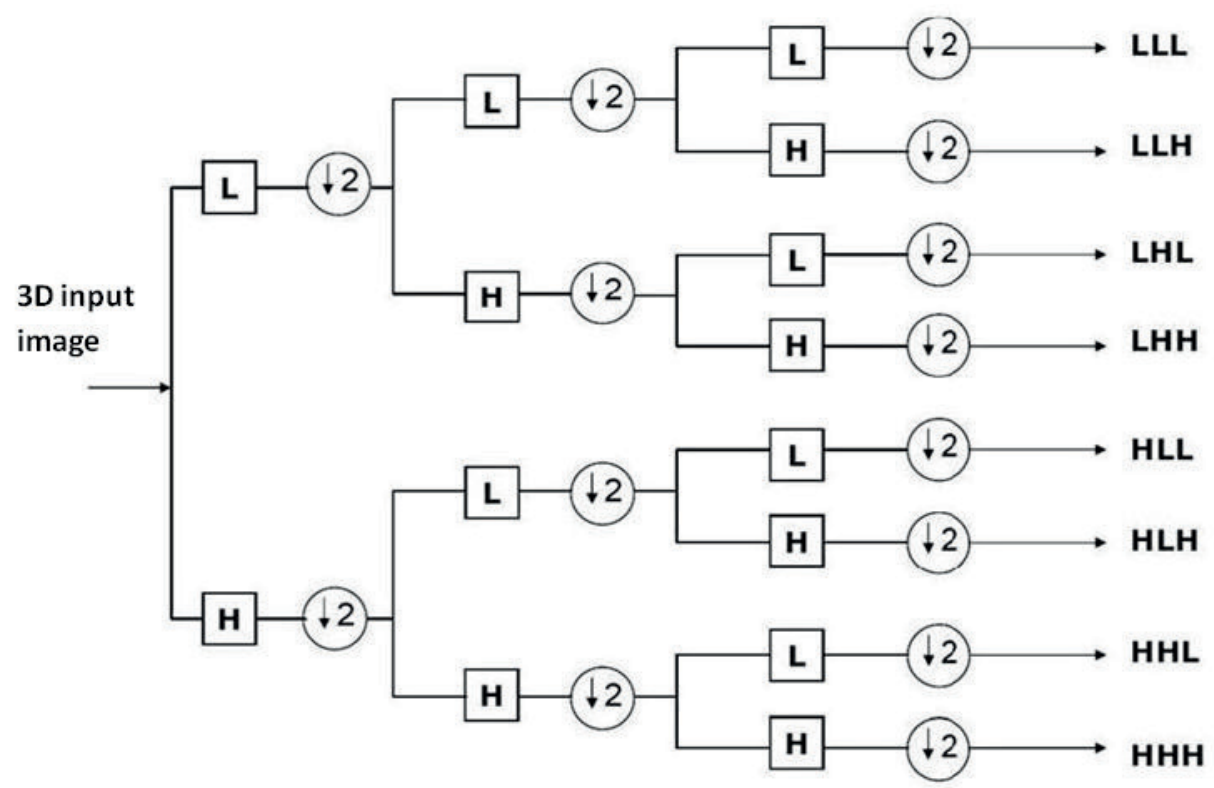

(12) downsampling

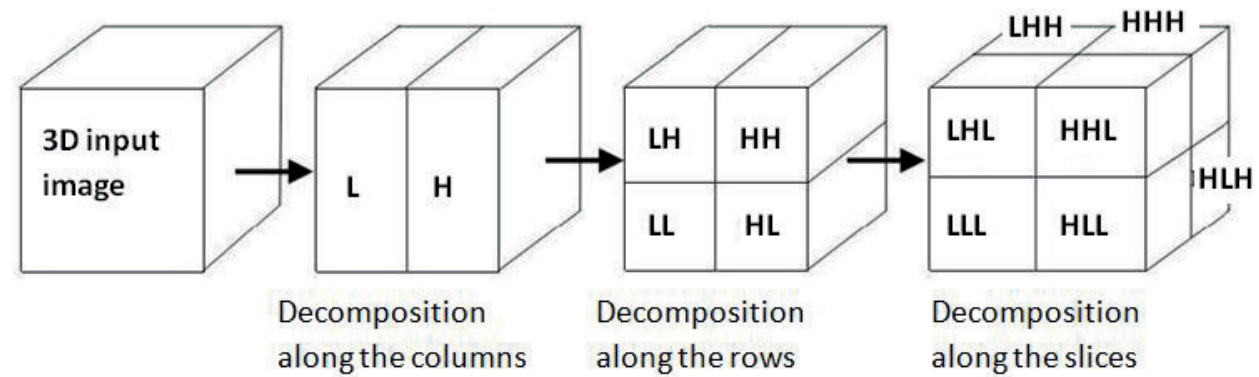

Fig. 3. 3D wavelet transform scheme

decomposed sub-images: LL, LH, HL and HH. This way the decomposition a 2D image is achieved. However, if we want to process a $3 \mathrm{D}$ image, then each of these four sub-images is filtered along the slices, resulting in eight sub-images: LLL, LLH, LHL, LHH, HLL, HLH, HHL and HHH [31]. The result of the two- or three-dimensional discrete wavelet transform is used as feature vector in face recognition process, as in the experiment. Visualisation of the feature data is shown in Figs. 4 and 5. The input data was quantized into slices and submitted to wavelet decomposition process.

In wavelet analysis, there are many types of wavelet functions which may be used for decomposition, and different function will produce different results for the same signal. Wavelet functions are characterized by properties such as orthogonality, symmetry and damping time. These properties are considered when choosing the function. Thus, the similarity between a signal and wavelet function are considered in face recognition, because better suited function brings out the features used for identification more effectively. In our method, we used $d b 1$ function for $2 \mathrm{D}$ images and $d b 10$ for $3 \mathrm{D}$ images. The functions were selected in experiments [32]. The experiment was carried

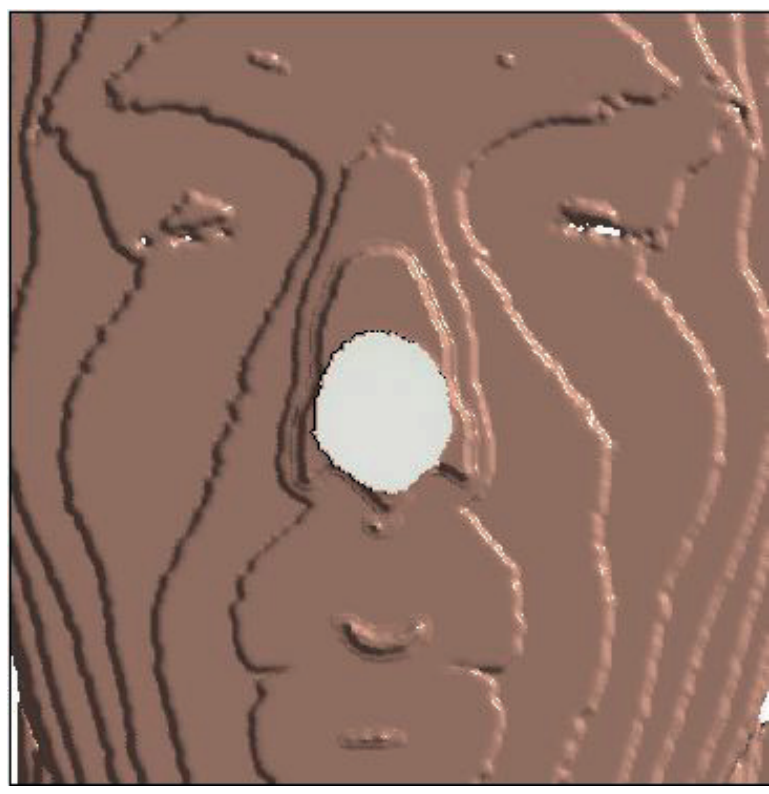

Fig. 4. 3D wavelet data visualization 


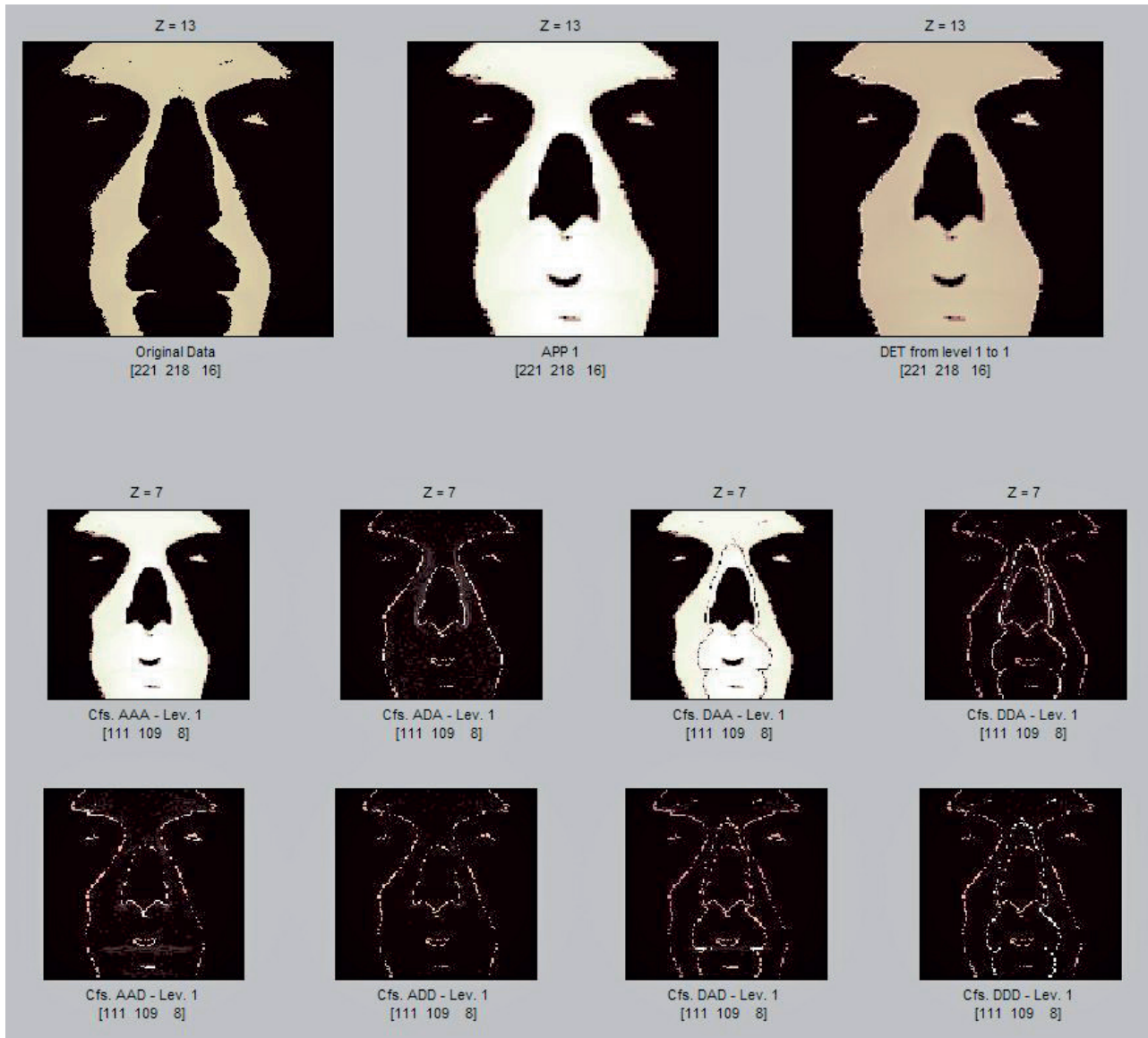

Fig. 5. 3D wavelet data visualization for one slice

out based on the UMB-DB face database [35]. The best results from among accessible wavelet functions were achieved for $d b 1$ function (2D data) and $d b 10$ (3D data) . In order to identify the best wavelet function, the method presented in this paper was applied. The results of experiments are presented in Table 1.

2.4. 2DHMM for face recognition. Classic HMM is a double stochastic process with underlying stochastic process that is not observable (hidden), but can be observed through another

Table 1

Comparison of recognition rate for different wavelet function

\begin{tabular}{l|c|c}
\hline Wavelet function & 2D Data & 3D Data \\
\hline \hline $\mathrm{db} 1$ & 93 & 86 \\
$\mathrm{db} 2$ & 90 & 88 \\
$\mathrm{db} 5$ & 88 & 92 \\
$\mathrm{db} 10$ & 86 & 94 \\
coif1 & 84 & 86 \\
coif5 & 74 & 76 \\
Sym2 & 81 & 83 \\
Sym5 & 79 & 75 \\
Bior 1.1 & 73 & 71 \\
Bior 2.6 & 71 & 67 \\
Bior 5.5 & 68 & 63 \\
\hline
\end{tabular}

set of stochastic processes producing a sequence of observation. Two-dimensional hidden Markov models (2DHMM) are a development of one-dimensional HMM and a reasonable method of two-dimensional data modelling for images. Yujian [23] proposed the definition and concept of 2DHMM, but presented solutions to these problems were adjusted to input data transformed to one-dimensional vectors. They were not fully satisfactory, since part of the information contained in the input signal was lost, and this data may be useful in the recognition systems. For signals such as images, their structure, that is, the relative position of pixels, is important to provide information. As a result of the analysis of this problem, we decided to develop a solution for the use of two-dimensional input data in two-dimensional Markov models and also extend it to 3D data.

The statistical parameters of the 2D model (Figs. 6, 7):

- size of model $N$

- number of states of the model $N^{2}$

- number of data streams $k_{1} \times k_{2}=K$

- number of $M$ symbols

- $i, j-$ indexes of states

- $l$ - the number of state

- the transition probabilities of the underlying Markov chain, $A=\left\{a_{i j l}\right\}, 1 \leq i, j \leq N, 1 \leq l \leq N^{2}$, where $a_{i j}$ is the probability of transition from state $i j$ to state $l$ 


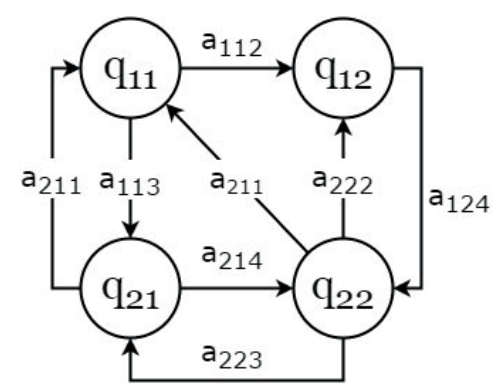

Fig. 6. Two-dimensional ergodic HMM

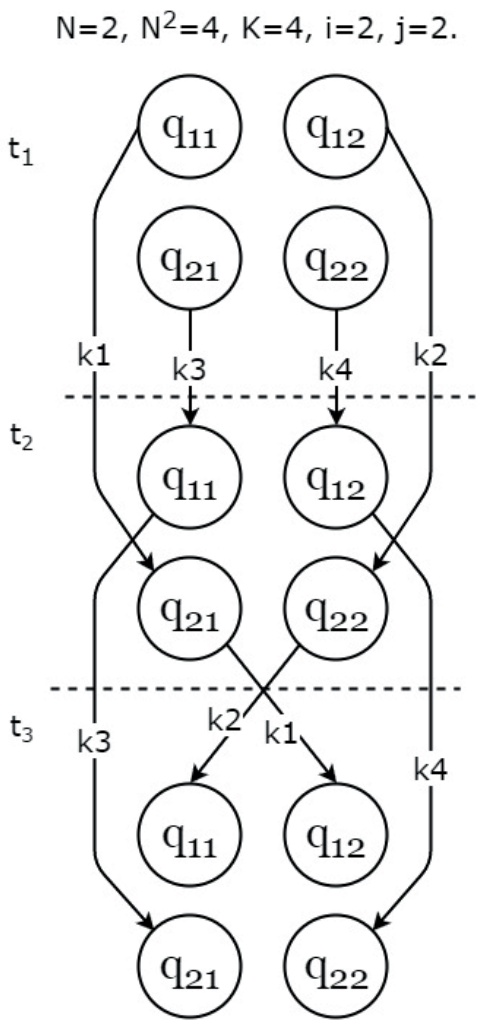

Fig. 7. The idea of 2DHMM

- the observation probabilities, $\left.B=\left\{b_{i j m}\right)\right\}, 1 \leq i, j \leq N, 1 \leq$ $\leq m \leq M$ which represent the probability of generating the $m_{\text {th }}$ symbol in the $i j_{\text {th }}$ state

- the initial probability, $\Pi=\left\{\pi_{i j k}\right\}, 1 \leq i, j \leq N, 1 \leq k \leq K$

- observation sequence, $O=\left\{o_{t}\right\}, 1 \leq t \leq T, o_{t}$ is a square matrix for simple observation with size $k_{1} \times k_{2}=K$.

In the proposed structure of the 2DHMM, $K$ processes operate parallelly for simplicity $K=N^{2}$. They share B matrix observation probabilities of generating and are dependent on each other. This feature distinguishes this solution from pseudo-2DHMM solutions, since we can apply 2D data as well as 3D data after dividing into slices.

Similarly to one-dimensional HMM, there are two fundamental problems of interest that must be solved for 2DHMM to be useful in face recognition applications. These problems are the following:

1. Given observation $O=\left(o_{1}, o_{2}, \ldots, o_{T}\right)$ and model $\lambda=(A$, $B, \Pi)$ that efficiently compute $P(O \mid \lambda)$

2. Given observation $O=\left(o_{1}, o_{2}, \ldots, o_{T}\right)$, estimate model parameters $\lambda=(A, B, \Pi)$ that maximize $P(O \mid \lambda)$

\subsection{Solution to Problem 1.}

The modified forward algorithm.

- Define forward variable $\alpha_{t}(i, j, k)$ as [33]:

$$
\alpha_{t}(i, j, k)=P\left(o_{1}, o_{2}, \ldots, o_{t}, q_{t}=i j \mid \lambda\right)
$$

- $\alpha_{t}(i, j, k)$ is the probability of observing the partial sequence $\left(o_{1}, o_{2}, \ldots, o_{t}\right)$ such that the state $q_{t}$ is $i, j$ for each $k_{t h}$ stream of data

- Induction

$$
\alpha_{t+1}(i, j, k)=\left[\sum_{l=1}^{N} \alpha_{t}(i, j, k) a_{i j l}\right] b_{i j}\left(o_{t+1}\right)
$$

\subsection{Solution to Problem 2.}

The modified parameters re-estimation algorithm.

- Define $\xi(i, j, l)$ as the probability of being in state $i j$ at time $t$ and in state $l$ at time $t+1$ for each $k_{t h}$ stream of data [33]:

$$
\begin{aligned}
\xi_{t}(i, j, l) & =\frac{\alpha_{t}(i, j, k) a_{i j l} b_{i j}\left(o_{t+1}\right) \beta_{t+1}(i, j, k)}{P(O \mid \lambda)}= \\
& =\frac{\alpha_{t}(i, j, k) a_{i j} b_{i j}\left(o_{t+1}\right) \beta_{t+1}(i, j, k)}{\sum_{k=1}^{K} \sum_{l=1}^{N^{2}} \alpha_{t}(i, j, k) a_{i j l} b_{i j}\left(o_{t+1}\right) \beta_{t+1}(i, j, k)}
\end{aligned}
$$

- Define $\gamma(i, j)$ as the probability of being in state $i, j$ at time $t$, given observation sequence.

$$
\gamma_{t}(i, j)=\sum_{l=1}^{N^{2}} \xi_{t}(i, j, l)
$$

- $\sum_{t=1}^{T} \gamma_{t}(i, j)$ is the expected number of times state $i, j$ is achieved

- $\sum_{t=1}^{T-1} \xi_{t}(i, j, l)$ is the expected number of transition from state ij to $l$ Update rules:

- $\pi_{i}^{-j} k=$ expected frequency in state $i, j$ at time $(t=1)=\gamma_{1}(i, j)$

- $\bar{a}(i, j)=($ expected number of transition from state $i, j$ to state $l$ )/(expected number of transitions from state $i, j$ :

$$
\bar{a}_{i j l}=\frac{\sum_{t} \xi_{t}(i, j, l)}{\sum_{t} \gamma_{t}(i, j)}
$$

- $b_{i j}(k)=($ expected number of times in state $j$ and observing symbol $k$ ) $/($ expected number of times in state $j$ :

$$
\bar{b}_{i j}(k)=\frac{\sum_{t, o_{t}=k} \gamma_{t}(i, j)}{\sum_{t} \gamma_{t}(i, j)}
$$




\section{Experiments}

In the experiments three image databases were used: FERET, UMB-DB and FRGC. We used FERET and UMB-DB databases for $2 \mathrm{D}$ data and for 3D data - UMB-DB and FRGC.

The Face Recognition Technology Program is managed by the Defense Advanced Research Projects Agency (DARPA) and the National Institute of Standards and Technology (NIST). As the result of this program, a large database of facial images gathered independently from algorithm developers was established. The database contains 1564 sets of images for a total of 14,126 images that includes 1199 individuals and 365 duplicate sets of images. A duplicate set is a second set of images of a person already in the database and was usually taken on a different day. For some individuals, over two years had passed between their first and last sessions, with some subjects being photographed multiple times [34].

The University of Milano Bicocca 3D face database (UMB-DB) is the set of multimodal (3D and 2D) colourful face images. 1473 images of 143 persons (30\% female and $70 \%$ male) are stored. Photos of faces were obtained in different lighting conditions and show the faces in different sizes and with different rotation angle [35]. The base is available for scientists and researchers interested in face biometrics.

As reported in [7], the FRGC database consists of 50,000 recordings divided into two partitions: training and validation. The validation partition consists of data from 4,000 subject sessions. A subject session is the set of all images of a person taken each time a person's biometric data is collected and consists of 4 controlled still images, 2 uncontrolled still images, and one three-dimensional image. The controlled images are full frontal facial images taken inside in a studio setting under two different sets of lighting conditions. The uncontrolled images were taken in varied illumination conditions. The 3D image was taken under controlled illumination conditions and consist of both a range and a texture image.

In order to verify the method, we chose randomly 100 persons as the data set, and for each individual we chose two random images for learning and two for testing. The size of each $2 \mathrm{D}$ image is $640 \times 480$ pixels, with 24 bits color. During pre-processing the image was converted to 256 gray levels per pixel, and after normalization selected area of face was resized to $220 \times 220$ pixels. $2 \mathrm{D}$ wavelet transform of second level was chosen as feature extraction technique for 2D data, and $d b 10$ as wavelet function. The size of feature vector was 48400 elements. For 3D data, 3D wavelet transform of first level was chosen, with $d b 1$ wavelet function. The 2D HMM implemented with parameters $N=4 ; N^{2}=16 ; K=16 ; M=25$. The parameters were chosen experimentally and the best results of recognition rate were obtained for those values. In the first experiment, the proposed 2DHMM was tested with 2D data for $2 \mathrm{D}$ face recognition algorithm. The $2 \mathrm{D}$ face images were chosen as learning and testing images. The procedure for $2 \mathrm{D}$ face recognition base on the forward algorithm is explained in Section 3.1. The 2D face image input is selected as testing data and is represented by the observation matrix $O$, obtained from the $2 \mathrm{D}$ wavelet transform. Next, each $2 \mathrm{DHMM}$ stored in the database was tested with observation matrix $O$, and the person for whom the sum of probability was maximum was chosen as the correct one. The probability of generating sequences of observations was computed from (3). Table 2 reports the results of this experiment with FERET and UMB-DB databases. The results obtained with our method were compared to other popular methods using the same databases. The average recognition rate of the method is $94 \%$, which is satisfactory in comparison to other methods. Good results of the first experiment encouraged us to carry out more experiments.

Table 2

Comparison of recognition rate $-2 \mathrm{D}$ face images [5]

\begin{tabular}{l|l|cc}
\hline Method & Databases & $\begin{array}{c}\text { Number } \\
\text { of images }\end{array}$ & $\begin{array}{c}\text { Recognition } \\
\text { rate [\%] }\end{array}$ \\
\hline \hline PCA & AR-Faces & 100 & 70 \\
LDA & AR-Faces & 100 & 88 \\
ICA & FERET & 200 & 89 \\
NN & FERET & 200 & 99 \\
PCA & UMB-DB & 90 & 94 \\
GWT+HMM & ORL & 40 & 99 \\
E-HMM & CMU & 68 & 99 \\
HMM+wavelet & ORL & 40 & 100 \\
1D HMM & UMB-DB & 100 & 90 \\
2D HMM our & UMB-DB & 200 & 93 \\
2D HMM our & FERET & 200 & 95 \\
\hline
\end{tabular}

The second experiment was performed with 3D data. The experiment procedure was similar to the previous one $-3 \mathrm{D}$ wavelet transform was used for features extraction. Table 3 reports the results of experiment with $3 \mathrm{D}$ data. The experiment was conducted on UMB-DB and FRGC databases, and the results were compared to other known methods. The recognition rate of the proposed technique for 3D data reached $94 \%$ for both databases, which is similar to PCA, but better than ICP.

Table 3

Comparison of recognition rate - 3D face images [6]

\begin{tabular}{l|l|c|c}
\hline Method & Databases & $\begin{array}{c}\text { Number } \\
\text { of images }\end{array}$ & $\begin{array}{c}\text { Recognition } \\
\text { rate [\%] }\end{array}$ \\
\hline \hline PCA & FRGC v1 & 276 & 95 \\
multi-ICP & FRGC v2 & 466 & 92 \\
Hausdorf distance & FRGC v1 & 200 & 98 \\
ICP+Gab+LBP & FRGC v1 & 200 & 99 \\
Deformable model & FRGC v2 & 466 & 90 \\
2D HMM our & UMB-DB & 200 & 94 \\
2D HMM our & FRGC v2 & 200 & 94 \\
\hline \multicolumn{2}{|l|}{}
\end{tabular}

The next experiment was conducted with multimodal data $(2 \mathrm{D}+3 \mathrm{D})$. The third test mostly combines the first two. Sepa- 


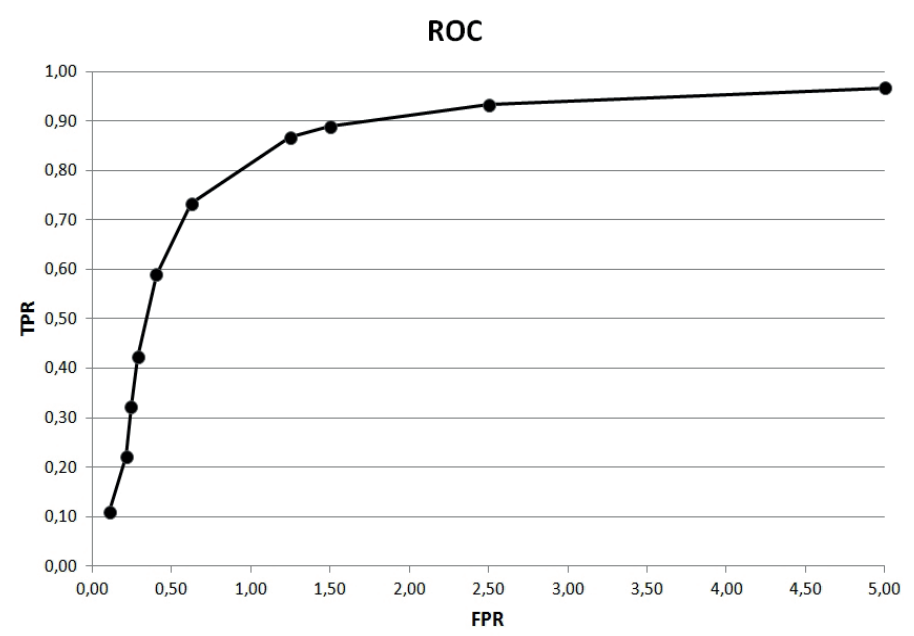

Fig. 8. ROC

rate models were created for $2 \mathrm{D}$ and $3 \mathrm{D}$ data. The sum of the generation of observation probabilities was used for deciding on the recognition. The results of this experiment are presented in Table 4 and compared to other popular methods. In this case, the recognition rate of the method proposed was $96 \%$ both for UMB-DB and FRGC, which is higher than the well-known ICP technique, but not as good as PCA. ROC of our method is shown in Fig. 8.

Table 4

Comparison of recognition rate $-2 \mathrm{D}+3 \mathrm{D}$ face images [6]

\begin{tabular}{l|l|c|c}
\hline Method & Databases & $\begin{array}{c}\text { Number } \\
\text { of images }\end{array}$ & $\begin{array}{c}\text { Recognition } \\
\text { rate [\%] }\end{array}$ \\
\hline \hline Graph match & FRGC v2 & 466 & 93 \\
ICP & FRGC v2 & 466 & 91 \\
PCA & $?$ & 200 & 99 \\
2D HMM our & UMB-DB & 200 & 96 \\
2D HMM our & FRGC v2 & 200 & 96 \\
\hline
\end{tabular}

Table 5

Comparison of recognition time

\begin{tabular}{l|c}
\hline Method & time $[\mathrm{s}]$ \\
\hline \hline ICP & 1 \\
2D HMM our & 0.7 \\
\hline
\end{tabular}

\section{Conclusion}

This study presents a new concept of true ergodic (not only left-right) two-dimensional hidden Markov models working with two-dimensional data. We have shown solutions of principle problems for ergodic 2DHMM, which may be applied for multimodal 2D and 3D data. The presented method allows for faster face processing and recognition because there is no need to change two- or three-dimensional input data of the image into a one-dimensional vector. Additionally, we saved the information included in the image structure. The obtained results are good as compared to other methods so the new method may be applied as an alternative solution. Experiments confirmed the validity of the concept of two-dimensional hidden Markov models. Furthermore, 2DHMM is a faster method as compared to ICP approach, in which it takes 1 second to compare two faces [4], while our method requires only $0.7 \mathrm{~s}$ (Table 4), which constitutes a significant advantages when working with a big database.

The research results indicate that face recognition with 3D images provides an alternative way of person identification. Unfortunately, 3D face recognition methods have their own issues and challenges, such as difficulties with data aquisition. Often there are problems with obtaining the full structure of 3D data and 2D data. Moreover, 3D images are not characterized by full illumination invariance, as many researchers claimed in the past. The experimental results confirmed that combined 2D and 3D multimodal data ensures better results than each sort individually.

\section{REFERENCES}

[1] W. Zhao, R. Chellappa, P. Phillips, A. Rosenfeld, "Face recognition: A literature survey", ACM Computing Surveys 35(4), 99-458 (2003).

[2] A. Rama, F. Tarres, J. Rurainsky, "Aligned texture map creation for pose invariant face recognition”, Multimedia Tools Applications 49(3), 545-565 (2010).

[3] K.I. Chang, K.W. Bowyer, “An evaluation of multimodal 2D+3D face biometrics", IEEE Transaction on Pattern Analysis and Machine Intelligence 27, 619-624 (2005).

[4] M.H. Mahoor, M. Abdel-Mottaleb, "Face recognition based on $3 \mathrm{~d}$ ridge images obtained from range data", Pattern Recognition 42(3), 445-451 (2009).

[5] A.F. Abate, M. Nappi, D. Riccio, G. Sabatino, "2D and 3D face recognition: A survey”, Pattern Recognition Letters 28(14), 1885-1906 (2007).

[6] K.W. Bowyer, K. Chang, P.J. Flynn, "A survey of approaches and challenges in $3 \mathrm{~d}$ and multi-modal $3 \mathrm{~d}+2 \mathrm{~d}$ face recognition", Computer Vision Image Understanding, 101, 1-15 (2006).

[7] P.J. Phillips, P.J. Flynn, T. Scruggs, K.W. Bowyer, J. Chang, K. Hoffman, J. Marques, J. Min, W. Worek, "Overview of the face recognition grand challenge", IEEE Conference on Computer Vision and Pattern Recognition, 947-954 (2005).

[8] X. Lu, A.K. Jain, "Deformation analysis for 3d face matching", 7th IEEE Workshop on Applications of Computer Vision, 99- 104 (2005).

[9] K.I Chang, K.W. Bowyer, P.J. Flynn, P.J., “Adaptive rigid multi-region selection for handling expression variation in $3 \mathrm{D}$ face recognition", IEEE Workshop on Face Recognition Grand Challenge Experiments, 157-164 (2005).

[10] G. Passalis, I. Kakadiaris, T. Theoharis, G. Toderici, N. Murtuza, "Evaluation of $3 \mathrm{~d}$ face recognition in the presence of facial expressions: an annotated deformable model approach", IEEE Workshop on Face Recognition Grand Challenge Experiments, 3, 171-179 (2005). 
[11] X. Lu, A.K. Jain, D. Colbry, "Matching 2.5D face scans to 3D models", IEEE Transactions on Pattern Analysis and Machine Intelligence 28(1) 31-43 (2006).

[12] G. Pan, S. Han, Z. Wu, Y. Wang, "3D face recognition using mapped depth images", IEEE Workshop on Face Recognition Grand Challenge Experiments, 175-181 (2005).

[13] B. Achermann, X. Jiang, H. Bunke, "Face recognition using range images", International Conference on Virtual Systems and MultiMedia, 129-136 (1997).

[14] A.B. Moreno, A. Sanchez, J.F. Velez, F.J. Diaz, "Face recognition using 3D local geometrical features: PCA vs. SVM", 4th International Symposium on Image and Signal Processing and Analysis, 185-190 (2005)

[15] A.B. Moreno, A. Sanchez, J.F. Velez, "Voxel-based 3D face representations for recognition", 12th International Workshop on Systems, Signals and Image Processing, 285-289 (2005).

[16] G. Gordon, "Face recognition based on depth and curvature features", Computer Vision and Pattern Recognition 1, 808-810 (1992).

[17] H.T. Tanaka, M. Ikeda, H. Chiaki, "Curvature-based face surface recognition using spherical correlation principal directions for curved object recognition", 3rd International Conference on Automated Face and Gesture Recognition, 372-377 (1998).

[18] J.A. Cook, V. Chandran, C. Fookes, "3D face recognition using log-Gabor templates", 17th British Machine Vision Conference, 769-778 (2006)

[19] Y. Jin, Y. Wang, Q. Ruan and X. Wang, "A new scheme for 3D face recognition based on 2D Gabor wavelet transform plus LBP," 6th International Conference on Computer Science \& Education, Singapore, 860-865 (2011).

[20] S. Berretti, A. Bimbo, P. Pala, "Automatic facial expression recognition in real-time from dynamic sequences of 3D face scans", The Visual Computer 29, 1333-1350 (2013).

[21] S. Eickeler, S. Mller, G. Rigoll, "High performance face recognition using pseudo 2-D hidden Markov models", European Control Conference, available at: http://citeseer.ist.psu.edu (1999).

[22] V. Bevilacqua, L. Cariello, G. Carro, D. Daleno, G. Mastronardi, "A face recognition system based on pseudo 2DHMM applied to neural network coefficients", Soft Computing, 12(7) 615-621 (2008).

[23] L. Yujian, "An analytic solution for estimating two-dimensional hidden Markov models", Applied Mathematics and Computation, $185,810-822$ (2007).
[24] J. Li, A. Najmi, R.M. Gray, "Image classification by a two dimensional hidden Markov model”, IEEE Transactions on Signal Processing, 48, 517-533 (2000).

[25] R. Kindermann, J.L. Snell, Markov Random Fields and their Applications, American Mathematical Society, Providence, Rhode Island (1980).

[26] M. Srinivasan and N. Ravichandran, "A new technique for face recognition using 2D-Gabor wavelet transform with 2D hidden Markov model approach", International Conference on Signal Processing, Image Processing \& Pattern Recognition, Coimbatore, 151-156 (2013).

[27] M. Bicego, U. Castellani and V. Murino, "Using hidden Markov models and wavelets for face recognition", Proceedings of the 12th International Conference on Image Analysis and Processing, 52-56 (2003).

[28] P.H. Lee, Y.W. Wang, J. Hsu, M.H. Yang, Y.P. Hung, "Robust facial feature extraction using embedded hidden Markov model for face recognition under large pose variation," Conference on Machine Vision Applications, Tokyo, 392-395 (2007).

[29] J. Bobulski, M. Kubanek, "Person identiffication system using an identikit picture of the suspect", Optica Applicata 42(4) 865-873 (2012).

[30] I. Daubechies, "The wavelet transform, time-frequency localization and signal analysis", IEEE Transaction on Information Theory, 36, 961-1005 (1990).

[31] A. Prochazka, L. Grafova, O. Vysata, "Three-dimensional wavelet transform in multi-dimensional biomedical volume processing", Proc. of the IASTED International Conference on Graphics and Virtual Reality, Cambridge, 263-268 (2011).

[32] J. Bobulski, "Wavelet transform in face recognition", Proceedings of 12th International Multi Conference on Advanced Computer Systems, Elk, 23-29 (2006).

[33] J. Bobulski, "2DHMM-based face recognition method", Image Processing \& Comunication Chalenges 7, 389, 11-18 (2016)

[34] P.J. Phillips, H. Moon, P.J. Rauss, S. Rizvi, "The FERET evaluation methodology for face recognition algorithms", IEEE Transactions on Pattern Analysis and Machine Intelligence, 22 (10), 1090-1104, (2000).

[35] A. Colombo, C. Cusano, R. Schettini, "UMB-DB: A database of partially Occluded 3D faces", Proc. IEEE International Conference on Computer Vision Workshops, Barcelona, 2113-2119, (2011). 